\title{
Biological agents in the treatment of uveitis
}

\author{
Maria Pia Paroli ${ }^{1}$, Alessandro Abbouda ${ }^{1}$, Irene Abicca ${ }^{1}$, Alfredo Sapìa ${ }^{1}$, Marino Paroli ${ }^{2}$ \\ ${ }^{1}$ Ocular Immunovirology Service, Department of Ophthalmology, Sapienza University, Rome, Italy \\ ${ }^{2}$ Clinical Immunology and Rheumatology, Department of Medical-Surgical Sciences and Biotechnologies, Sapienza University, \\ Latina, Italy \\ Email: mariapia.paroli@uniroma1.it
}

Received 21 May 2013; revised 23 June 2013; accepted 15 July 2013

Copyright (C) 2013 Maria Pia Paroli et al. This is an open access article distributed under the Creative Commons Attribution License, which permits unrestricted use, distribution, and reproduction in any medium, provided the original work is properly cited.

\begin{abstract}
Inflammatory uveitis is a difficult condition to treat. Recently, a new class of drugs obtained by a biological process and therefore defined as "biologics" has been successfully used in the treatment of immunemediated rheumatic diseases. These drugs target different pro-inflammatory cytokines including tumor necrosis factor- $\alpha$, interleukin-1 and interleukin-6, or immune effector cells including $B$ and $T$ lymphocytes. Their use has been then extended to different forms of uveitis resistant to standard therapy in small uncontrolled trials, with general and unexpected efficacy. The present review analyzes the literature on treatment of uveitis with the biologics currently available for the treatment of inflammatory rheumatic diseases. We used PubMed search engine as the main source of information. Among the papers included in the reference list, a particular emphasis was given on articles published during the last 5 years.
\end{abstract}

Keywords: Biologics; Uveitis

\section{INTRODUCTION}

In severe endogenous uveitis, there is a significant risk of visual loss [1]. Although the standard treatment based on the use on steroid and immunosuppressant is often effective in preventing this irreversible complication, side effects or lack of treatment efficacy may require a different therapy. The advent of so-called "biologics" which are indicated for the treatment of inflammatory rheumatic diseases has added additional opportunities in dealing with resistant or recurrent cases of chronic non-infectious uveitis [2,3]. However, these drugs are much expensive and are not devoid of clinically relevant adverse effects [4]. Furthermore, the effectiveness of these agents has been found to vary greatly depending on the individual case treated. In addition, lack of randomized clinical trials due to the relative rarity of inflammatory uveitis has a significant negative effect, namely treatment decisions by the physician are made only on personal experience, on published uncontrolled observations or are influenced by expert opinions. In this review, we analyzed the most relevant and recent data on the use of the single biologics for the treatment of the several forms of inflammatory uveitis. We used PubMed search engine as the main source of information, with a special accent on articles published during the last 5 years. This review therefore represents a literatures summary about the single biologics for the treatment of the several forms of inflammatory uveitis.

\section{RESULTS FROM STUDIES ON THE INDIVIDUAL BIOLOGICS}

\subsection{Infliximab}

Infliximab is a chimeric human-mouse monoclonal antibody against tumor necrosis factor (TNF)- $\alpha$. The standard dose used is $3-5 \mathrm{mg} / \mathrm{kg}$ which can be raised up to $10 \mathrm{mg} / \mathrm{kg}$ i.v. infusions are generally repeated after 2 and 6 weeks and then after 8 weeks, but in patients with uveitis infusions are generally repeated more frequently and at a high dose up to $20 \mathrm{mg} / \mathrm{kg}$ [5]. Classical rheumatologic indications include moderate to severe rheumatoid arthritis (RA) in combination with methotrexate (MTX), moderate to severe psoriatic arthritis (PA) which has not responded to conventional DMARDs and severe active ankylosing spondylitis (AS) after an inadequate response to conventional therapy. Infliximab is also indicated for the treatment of bowel inflammatory diseases including Crohn's disease and ulcerative colitis. Infusion reactions to infliximab are rare but can be severe. Moreover, infliximab can induce anti-drug antibodies which can interfere or even abolish the drug effectiveness [6]. As other anti-TNF- $\alpha$ agents, treatment with infliximab is associated with a significant increase of infections, including 
tuberculosis, bacterial, viral and parasite infections [7]. In 2001, an initial study on the efficacy of anti-TNF- $\alpha$ agents on inflammatory eye diseases was published by Smith et al. who showed that both infliximab might benefit certain subgroups of patients with inflammatory eye disease [8]. The same year, it was reported by the journal The Lancet that after treatment with infliximab of a series of patients with Behçet's disease, remission of ocular inflammation was already evident within the first $24 \mathrm{~h}$, and complete suppression was seen after 7 days [9]. Several studies have been then carried out to confirm the efficacy of infliximab in Behçet's disease [10-17]. Recently, in a large multicenter study, the effects of infliximab on refractory uveoretinitis in patients with Behçet's disease during the first year of treatment was evaluated [18]. A total of 63 patients were studied. The efficacy analysis at 1 year showed that uveoretinitis had improved in $69 \%$, had improved somewhat in $23 \%$, was unchanged in $8 \%$, and had worsened in no patients. The mean number of ocular attacks per 6-month period decreased from 2.66 at baseline to 0.44 during months 1 through 6 of infliximab therapy and to 0.79 during months 7 through 12. Efficacy was best for patients with uveoretinitis duration of less than 5 years. At the end of 1 year, uveoretinitis had improved in $92 \%$ of patients, accompanied by improvement in the mean visual acuity (VA). In another recent study, long-term efficacy of infliximab was evaluated in patients with refractory Behçet's disease-associated and idiopathic posterior uveitis (PU) [19]. The single center study involved 50 consecutive patients who had failed at least one immunosuppressive drug. At the final 6-year follow-up, mean both right and left eye (VA) increased significantly from basal levels. A complete response of PU was recorded in $68 \%$ patients and partial response in $22 \%$. Five patients were non-responders and withdrew from the study after the third infusion. This study further confirmed that long-term infliximab therapy was effective and safe with a significant VA gain in refractory Behçet's diseaseassociated and idiopathic PU. In other studies, however, it has reported a possible resistance to infliximab activity due to anti-drug antibodies that can impair in some cases the efficacy of the drug [20,21]. Moreover, during therapy with infliximab of Behçet's disease-associated uveitis, side effects have been found to be relatively rare, but may be serious. Moreover, although the last reports appears quite optimistic, there are still conflicting data as to the ability of the drug to induce durable remission after cessation of treatment [22].

The use of infliximab has been also tested for the treatment of other types of chronic non-infectious uveitis. The efficacy of infliximab was studied in uveitis associated with Crohn's disease and spondyloarthropathy with good results [23-27]. Different studies demonstrated that infliximab is useful in the treatment of uveitis associated with polyarticular juvenile idiopathic arthritis (JIA) non-responsive to standard therapy [28,29]. Infliximab treatment was found to be advantageous also in the treatment of Vogt-Koyanagi-Harada (VKH) syndrome, but only as adjunctive therapy [30]. Interestingly, in a retrospective study, it was found that although infliximab is beneficial in the treatment of uveitis, a dose escalation up to 4 times above the approved dose is often necessary to achieve disease control in patients with uveitis, and that doses even $>10 \mathrm{mg} / \mathrm{kg}$ every 4 weeks may not be sufficient to control the disease [31].

Quite recently, the safety and long-term effects of intravitreal infliximab on chronic noninfectious uveitis has been evaluated in 10 eyes of 7 patients [32]. About $1.5 \mathrm{mg}$ of infliximab in $0.15 \mathrm{~mL}$ was injected intravitreally, and the patients were followed for 6 months. Results showed that vision improved and the macular edema decreased, but the effect was temporary suggesting the need of repeated injections to achieve the best therapeutic goal. In another study, safety and efficacy of intravitreal infliximab were evaluated for sight-threatening relapsing uveitis in Behçet's disease [33]. A single intravitreal injection of infliximab $(1 \mathrm{mg} / 0.05 \mathrm{~mL})$ was given to 15 patients with relapsing PU at the onset of a unilateral attack. Baseline best-corrected VA improved significantly by day 7 and continued to improve through day 30 after infliximab, a profound decrease in anterior chamber cells and vitreous haze as well as beneficial effects in retinal vasculitis and retinitis were evident through day 30. Cystoid macular edema persisted in 9 of 11 eyes affected, but central macular thickness decreased. These findings suggest that in Behçet's disease intravitreal infliximab might be considered when systemic administration is not feasible or contraindicated.

\subsection{Adalimumab}

Adalimumab is a fully humanized anti-TNF- $\alpha$ monoclonal antibody that binds TNF- $\alpha$ as both soluble precursor and membrane bound protein. Adalimumab can induce lysis by apoptosis or cytotoxicity of the cells expressing this cytokine. The drug blocks the interaction between TNF and the cell surface receptors p55 and p75, and being highly specific for TNF does not inhibit the lymphotoxin TNF $\beta$. The usual dose is $40 \mathrm{mg}$ (in children $24 \mathrm{mg} / \mathrm{m}^{2}$ ) subcutaneously every two weeks. Therapeutic indications include moderate to severe RA in combination or not with MTX, moderate to severe PA which has not responded to DMARDs, in adults with severe active SA with inadequate responses to conventional therapy, Crohn's disease and ulcerative colitis in both adults and children who have not responded to standard therapy. A definitive evidence that adalimumab is an effective treatment in anterior uveitis came from a study involving 
1250 patients with active AS enrolled in a multinational, open-label, uncontrolled clinical study [34]. During adalimumab treatment, the rate of anterior uveitis (AU) flares was reduced by $51 \%$ in all patients, by $58 \%$ in 274 patients with a history of $\mathrm{AU}$, by $68 \%$ in 106 patients with a recent history of AU, by $50 \%$ in 28 patients with symptomatic AU at baseline and by $45 \%$ in 43 patients with chronic uveitis. Moreover, AU flares during adalimumab treatment were predominantly mild. Adalimumab has been found to be effective in Behçet's disease in several studies, with clinical remission of and absence of recurrence during treatment [35]. The usefulness of adalimumab is particularly evident for patient showing hypersensitivity to infliximab [36]. Long-term effects of adalimumab in the treatment of uveitis associated with JIA was assessed in another recent study [37]. Adalimumab was initiated in 54 patients with JIA to treat active arthritis and active associated uveitis. At the end of the study, uveitis was under good clinical control in two thirds of 54 patients and one third had active uveitis. According to SUN criteria, adalimumab treatment for uveitis showed improved activity in $28 \%$ of patients, with a moderate response in 16 patients, no change in a further 16 patients, and worsening activity in $13 \%$ of patients. The authors concluded that adalimumab is a valuable option in the treatment of uveitis associated with active JIA. Adalimumab has proven to be effective also in sarcoidosis-associated refractory chronic noninfectious uveitis [38]. In a study involving 26 patients, choroid involvement resolved in 10/15 patients, five had partial improvement; vasculitis resolved in $1 / 1$ patient; papillitis resolved in $7 / 8$ patients, one had partial response; macular edema resolved in $5 / 8$ patients, three had partial response; vitreous cleared completely in $5 / 5$ patients. Overall outcome regarding intraocular inflammatory signs showed improvement in 22 patients $(85 \%)$ and stabilization in four patients (15\%). At 12 months, no recurrences were reported in those successfully treated. All laboratory parameters of inflammatory In a recent prospective case series, a total of 131 patients with refractory uveitis and intolerance or failure to respond to prednisone and at least 1 other systemic immunosuppressive were treated with adalimumab [39]. Thirty-nine patients had JIA, 16 patients pars planitis 13 patients Behçet's disease, and with uveitis of idiopathic origin were studied. Anterior chamber inflammation and vitreous inflammation decreased significantly from a mean of 1.51 and 1.03 at baseline to 0.25 and 0.14 , respectively, at 6 months. Macular thickness improved as well as VA. Cystoid macular edema, which was present in 40 eyes at baseline, showed complete resolution in 28 eyes at 6 months. Six months after the initiation of the study, 111 patients $(85 \%)$ were able to reduce at least $50 \%$ of their baseline immuno-suppressant load. Only 9 patients $(6.9 \%)$ had severe relapses during the 6 months of follow-up. Therefore, adalimumab was well tolerated and helpful in decreasing inflammatory activity in refractory uveitis was able to reduce steroid requirement. Interestingly, adalimumab has proven to be an effective alternative in patients no more responsive or allergic to infliximab [40-42].

\subsection{Etanercept}

Etanercept is a fusion protein combining two p75 TNF receptors with an Fc fragment of human IgG1. The drug binds to both the TNF- $\alpha$ that TNF- $\beta$. It has the same indications as adalimumab with the exclusion of inflammatory bowel diseases. Usual dose is $50 \mathrm{mg}$ subcutaneously once weekly.

In pediatric rheumatology, etanercept is used at a dose of $0.8 \mathrm{mg} / \mathrm{kg}$ given once weekly.

In a study of 2001, 7 children with uveitis associated with pauciarticular JIA who had failed previous therapy with standard therapy were treated with Etanercept [43]. VA improved in $40 \%$ of eyes treated. However, an exacerbation of uveitis during etanercept therapy occurred in 1 child. These data suggested that etanercept had a beneficial effect on treatment-resistant chronic uveitis in children. Subsequently, several studies have been set up to assess the efficacy and safety of etanercept in the treatment of uveitis. It has been then reported that although most patients with JIA-associated uveitis initially respond to etanercept, this initial response is not always followed by sustained improvement over longer periods of time, and is associate with possible uveitis flare [44]. In a case report, a HLA-B27+ patient treated with etanercept for resistant AS developed uveitis, promptly reverted after etanercept withdrawal [45]. Conversely, in another study, in 6 of 10 children enrolled in a small, prospective open-label trial, etanercept had a beneficial effect at least 3 months, and 4 children exhibited sustained benefit for at least 1 year [46]. This suggested that etanercept might represent a viable alternative for children with active uveitis who have been treated unsuccessfully with traditional disease-modifying antirheumatic drugs. In another, it was observed that during treatment with etanercept, there were both relapses and first courses of uveitis. However, frequency and severity of uveitis seemed not to be influenced by etanercept [47]. In a comprehensive literature review, it has been reported that ocular inflammation is paradoxically a potential adverse event following the use of etanercept in both previously uninvolved and inflamed eyes [48]. Therefore, author concluded that careful surveillance of patients on etanercept is warranted to assure that flares in ocular inflammation are not secondary to etanercept therapy. Moreover, in another literature review analyzing data from 2 spontaneous reporting databases, there were 43 
cases of uveitis associated with etanercept, 14 associated with infliximab, and 2 associated with adalimumab. After normalizing for the estimated number of patients treated with each medication, etanercept was associated with a greater number of uveitis cases than infliximab and adalimumab while no such association was found between adalimumab and infliximab. Using a priori criteria to avoid inclusion of patients whose underlying disease was associated with uveitis, 20 cases of uveitis occurred during treatment associated with etanercept, 4 cases associated with infliximab, and 2 cases associated with adalimumab were identified. However, the authors commented that their findings do not support the use of infliximab over etanercept but, rather, if a patient develops uveitis during etanercept therapy, then a change to infliximab may be warranted [49]. In a very recent study, it has been observed from data reported in the literature that forty-two cases of inflammatory eye diseases believed to be associated with the use of etanercept include 33 cases of uveitis, 8 scleritis, 1 orbital myositis [50]. These data have been obtained from 16 patients with RA, 10 with JIA, 14 with AS, and 2 with psoriatic spondyloarthropathy. The final conclusion underlines that ocular inflammation is paradoxically a potential adverse effect of etanercept, even in previously uninvolved eyes, although it seems to involve a minority of the patients treated.

\subsection{Tocilizumab}

Tocilizumab is a fully humanized monoclonal antibody recognizing IL-6 receptor (IL-6 R) in both its soluble form or bound to cell membrane surface. Its indication includes moderate to severe RA. The standard dose is 8 $\mathrm{mg} / \mathrm{kg}$ month intravenously. Clinical trials have shown promising results in treating several autoimmune diseases in patients who respond inadequately to conventional immunosuppressant, including large-vessel vasculitis [51]. In the first report on the efficacy of tocilizumab in uveitis, a patient with birdshot retinochoroidopathy was first treated with adalimumab after failure of use of standard immunosoppressants [52]. However, no significant improvement was noted. Intravenous injections of tocilizumab with improvement of VA was noted in both eyes and remained stable after 8 months. In the same study, in a second patient who presented with acute panuveitis in the right eye a diagnosis of idiopathic uveitis was made. After treatment with corticosteroid treatment associated with methotrexate, azathioprine or mycophenolate mofetil, adalimumab was started with no improvement. Finally tocilizumab was administered $8 \mathrm{mg} / \mathrm{kg}$ imonthly i.v. with improvement of VA which remained stable after 6 monthly injections of tocilizumab, allowing tapering of prednisolone. In one case of neuro-Behçet's disease, a young patient with a ten year history of recurrent attacks of oral ulcers, chronic bilateral uveitis and cutaneous vasculitis was firstly treated with methotrexate, azathioprine, mycophenolate mofetil, cyclosporine, interferon, daclizumab, and cyclophosphamide with incomplete responses or unacceptable toxicity [53]. Doses of $30-60 \mathrm{mg} /$ day of prednisone were required to suppress headaches and fevers. Infliximab by intravenous infusion was then started with a high frequency regimen due to refractory severe uveitis. Disease manifestations were completely suppressed. After 63 infusions, he developed IgA nephropathy. Off-label treatment with tocilizumab was then initiated at a dose of $8 \mathrm{mg} / \mathrm{kg}$ intravenously every four weeks with partial response within days. By the time of the second infusion, he had total remission of symptoms and signs and had completely tapered off prednisone. The patient's last MRI was totally normal without any abnormal enhancement and no signs of uveitis. However, treatment was not effective in suppressing oral aphthae. Finally, three adult patients with JIA-associated chronic AU with presence of vision-threatening complications were treated with intravenous tocilizumab $8 \mathrm{mg} / \mathrm{kg}$ body weight at 4-weekly intervals [54]. All patients the disease had been refractory to high dosages of topical corticosteroids and previous systemic corticosteroid treatment and DMARD, including at least 1 TNF$\alpha$ inhibitor. Within the follow-up period under tocilizumab treatment (mean follow-up 9 months), inactivity of the uveitis was achieved in patients 2 and 3 uveitis continued in the other patient, requiring a further increase in the dosage of topical steroids. The authors conclude that tocilizumab may represent a treatment option for otherwise refractory JIA-associated uveitis, although further prospective studies are needed to evaluate the efficacy of this new drug in comparison to other biologics.

\subsection{Golimumab}

Golimumab is a fully humanized monoclonal antibody against TNF- $\alpha$ with the same indications of etanercept. Compared to other anti TNF- $\alpha$ antibodies, golimumab is characterized by light and heavy chains super imposable to those of humans with a consequent reduction of immunogenicity. Is administered subcutaneously and has the greater interval between administrations (28 days).

In a first study, 2 patients were treated. Patient 1 with JIA developed a severe AU in his right eye after therapy with etanercept. He switched to infliximab and secondly to adalimumab due to hypersensitivity reaction. He then underwent cataract surgery of his right eye and his uveitis relapsed. Adalimumab was the switched to golimumab in addition to MTX got both his uveitis and arthritis under control for at least seven months after initiation of treatment with golimumab [39]. Patient 2 diagnosed with idiopathic retinal vasculitis started with adalimumab that was then switched to golimumab plus MTX due to worsening of the disease. After 6 months of treatment with 
golimumab and MTX the patient remained asymptomatic and ophthalmic examination reveals no sign of active inflammation. In another study, 3 patients with JIA uveitis were treated with golimumab. Patient 1 was refractory to multiple medications but achieved long-term quiescence with golimumab. Patient 2 had an initial response to golimumab but then developed recurrence of both ocular and joint symptoms and have failed numerous other therapies. Patient 3 failed three TNF inhibitors before golimumab therapy was initiated with significant improvement in ocular inflammation [55]. The authors conclude that golimumab appears to be safe and provides an alternative treatment in refractory JIA uveitis, including patients who have failed other TNF inhibitors, although further studies are required to understand the long-term efficacy and safety of golimumab in the treatment of uveitis.

\subsection{Anti-IL-1 Antibodies}

Interleukin-1 has been proven to play an important role in experimental uveitis [56]. For this reason, antagonists of this cytokine have been tested in human uveitis. However, few data are available to date, and results are still inconclusive. In a first study, a 4 years old boy with CINCA syndrome and bilateral panuveitis not-responsive to standard immunosuppressive therapy was treated with etanercept [57]. However, over the following 2 years, further relapses of uveitis with disc swelling and vitritis associated with only partial improvement of his arthropathy occurred. Treatment with rHuIL1Ra (anakinra) was started at a dose of $1 \mathrm{mg} / \mathrm{kg} / \mathrm{day}$, inducing remission. At the age of 8 years old, his uveitis remained quiescent with resolved vitritis and disc swelling, and permitted withdrawal of oral prednisolone. Vision remained 6/9 in the right eye and 6/18 in the left, with normal intraocular pressures. Subsequently, an interesting study has been recently published on the effect of XOMA 052 (gevokizumab), a recombinant humanized anti-IL-1 $\beta$ antibody shown to modulate IL-1 $\beta$ activity, in 7 Behçet's disease patients with acute intraocular inflammation [58]. All patients experienced rapid and clinically meaningful improvements in VA and reductions in anterior chamber cells and flare, vitreous haze and retinal infiltrates in the index. Intraocular inflammation began to resolve in all patients on days $1-4$. Complete resolution of retinal findings was achieved in $4-21$ days. Another had hypopyon on day 0 , and it was almost completely resolved on day 1 following study drug administration, with a concomitant reduction in flare In the remaining five patients with anterior chamber cells, a clinically significant improvement was observed within 7 - 21 days. As conclusive results, in five of seven patients, intraocular inflammation was resolved fully without signs of new exacer- bation at day 28. Moreover, responses were durable, with a median duration of 49 days (range 21 - 97 days). Interestingly, a $97 \%$ less production of IL-1 $\beta$ as well as reduced production of IL- 6 and IL- $1 \alpha$ upon stimulation with lipopolysaccharide 28 days after the infusion was observed. It appears that after 28 days of neutralization of endogenous IL-1 $\beta$, the circulating white blood cells have developed a less inflammatory phenotype compared with baseline. In the same assay there was no reduction in the production of IL-1Ra, suggesting that prolonged neutralization of IL-1 $\beta$ at this concentration does not dampen the ability to produce this natural inhibitor of IL-1 activity. The authors conclude that XOMA 052 may represent a future effective treatment of uveitis and retinal vasculitis and other types of non-infectious inflammatory uveitis.

\subsection{Rituximab}

Rituximab is an anti-CD20 chimeric mouse-human IgG1 monoclonal antibody originally developed for the treatment of B cell lymphomas. The usual dose is $1000 \mathrm{mg}$ per infusion on day 1 and 15 and then at month 12 and 21. Rituximab is licensed also for RA, being particularly effective in subgroups of patients, which are positive for anti-citrullinated protein antibody and/or rheumatoid factor (seropositive RA). The binding and clustering of CD20 by rituximab transmits a signal that causes apoptosis of B-lymphocytes. Infusions of rituximab induce the depletion of B-lymphocytes from peripheral blood for several months. The drug has been used in clinical trials in different autoimmune diseases including systemic lupus erythematosus, Sjogren's syndrome ANCAassociated vasculitis [59-61]. Although inflammatory uveitis is believed to be a T-cell mediated disease, B-cell depletion may be effective since these cells are efficient antigen-presenting cells for T-lymphocytes and contribute to their activation and clonal expansion [62].

In a first study, a woman was suffering from bilateral, noninfectious chronic anterior uveitis refractive to corticosteroids and immunosuppressive drugs, was treated with rituximab [63]. After treatment with rituximab, vision and cystoid macular edema improved, and uveitis was stable until at least 12 months.

In another study 20 patients with Behçet's disease were treated with rituximab in comparison with other standard immunosuppressant. TADAI, edema of retina, disc and macula improved significantly better in the rituximab group. Adverse effects were observed mainly due to an increased risk of infection, especially in patients receiving concomitant therapy with corticosteroids and immunosuppressant [64]. Severe uveitis was also successfully treated with rituximab in 10 patients with JIA [65]. Rituximab appears therefore an appealing tool for 
treatment of chronic non-infectious uveitis, although further study is needed to clarify important aspects of both safety and efficacy [66].

\subsection{Abatacept}

Abatacept is a fusion protein composed of the Fc region of the immunoglobulin IgG1 fused to the extracellular domain of CTLA-4 molecule. Abatacept is a selective co-stimulation modulator as it inhibits the co-stimulation of T-Cells, preventing antigen-presenting cells (APCs) from delivering the co-stimulatory signal to $\mathrm{T}$ cells to fully activate them. It is usually administered intravenously at a dose ranging from 5000 to $1000 \mathrm{mg}$ every four weeks.

In a recent study 7 patients with JIA-related uveitis refractory to immunosuppressive and anti-TNF- $\alpha$ agents were treated with abatacept [67]. All patients responded to abatacept and 6 maintained a clinical remission after a mean of 9.2 months of treatment. The authors conclude that abatacept treatment may lead to sustained improvement in severe anti-TNF- $\alpha$-resistant JIA-related uveitis, providing new insights into a possible indication of abatacept for the treatment of uveitis.

\section{CONCLUSION}

A further understanding of the mechanisms underlying the pathogenesis of uveitis is crucial for set-up innovative treatments aimed to reduce inflammation and prevent severe ocular complications including visual loss. At present, the treatment of non-infectious uveitis with biologics represents a novel and very interesting opportunity. Targets of biologics include TNF- $\alpha$, IL-1, IL-6, B- and T-lymphocytes. Due to the cost of biologics and the possible infectious complications, their use is reserved for those cases, which do not respond adequately or are intolerant to standard treatment with corticosteroids and immunosuppressant. Among the single biologics, infliximab and adalimumab are the most studied agents, and show a consistent efficacy for the treatment of the different form of non-infectious uveitis. Etanercept, although paradoxically responsible in very rare cases of the onset of uveitis in patients with inflammatory rheumatic conditions, is still an effective drug for the treatment of inflammatory ocular diseases. Tocilizumab, golimumab, anakinra, rituximab and abatacept necessitate further study to ascertain their actual role in the therapy of uveitis. However, for all the biologics described here, prospective randomized controlled trials are urgently needed to define both benefits and risks of these agents in the treatment of the different types of uveitis, in order to help the clinicians to make the better decision about treatment of their patients, and to clarify the role of switching from a biologic to another in case of proven inefficacy of one or more of them.

\section{REFERENCES}

[1] Durrani, O.M., Meads, C.A. and Murray, P.I. (2004) Uveitis: A potentially blinding disease. Ophthalmologica, 21, 8223-8236.

[2] Szekanecz, Z., Szanto, S., Szabo, Z., Vancsa, A., Szamosi, S., Bodnar, N., et al. (2010) Biologics-beyond the joints. Autoimmunity Reviews, 9, 820-824. doi:10.1016/j.autrev.2010.07.011

[3] Bell, G.M., Reynolds, G. and Isaacs, J.D. (2011) Biologic therapies in non-rheumatic diseases: Lessons for rheumatologists? Nature Reviews Rheumatology, 7, 507-516. doi:10.1038/nrrheum.2011.106

[4] Hyrich, K.L., Watson, K.D., Isenberg, D.A. and Symmons, D.P. (2008) The British Society for Rheumatology Biologics Register: 6 years on. Rheumatology (Oxford), 47, 1441-1443. doi:10.1093/rheumatology/ken242

[5] Kahn, P., Weiss, M., Imundo, L.F. and Levy, D.M. (2006) Favorable response to high-dose infliximab for refractory childhood uveitis. Ophthalmology, 113, 860-864.

[6] Emi Aikawa, N., de Carvalho, J.F., Artur Almeida Silva, C. and Bonfa, E. (2010) Immunogenicity of Anti-TNFalpha agents in autoimmune diseases. Clinical Reviews in Allergy \& Immunology, 38, 82-89. doi:10.1007/s12016-009-8140-3

[7] Aaltonen, K.J., Virkki, L.M., Malmivaara, A., Konttinen, Y.T., Nordstrom, D.C. and Blom, M. (2012) Systematic review and meta-analysis of the efficacy and safety of existing TNF blocking agents in treatment of rheumatoid arthritis. PLoS One, 7, e30275. doi:10.1371/journal.pone.0030275

[8] Smith, J.R., Levinson, R.D., Holland, G.N., Jabs, D.A., Robinson, M.R., Whitcup, S.M., et al. (2001) Differential efficacy of tumor necrosis factor inhibition in the management of inflammatory eye disease and associated rheumatic disease. Arthritis \& Rheumatism, 45, 252-257. doi:10.1002/1529-0131(200106)45:3<252::AID-ART257 $>3.0 . \mathrm{CO}, 2-5$

[9] Sfikakis, P.P., Theodossiadis, P.G., Katsiari, C.G., Kaklamanis, P. and Markomichelakis, N.N. (2001) Effect of infliximab on sight-threatening panuveitis in Behçet's disease. Lancet, 358, 295-296. doi:10.1016/S0140-6736(01)05497-6

[10] Accorinti, M., Pirraglia, M.P., Paroli, M.P., Priori, R., Conti, F. and Pivetti-Pezzi, P. (2007) Infliximab treatment for ocular and extraocular manifestations of Behçet's disease. Japanese Journal of Ophthalmology, 51, 191-196. doi:10.1007/s10384-006-0425-y

[11] Tugal-Tutkun, I., Mudun, A., Urgancioglu, M., Kamali, S., Kasapoglu, E., Inanc, M., et al. (2005) Efficacy of infliximab in the treatment of uveitis that is resistant to treatment with the combination of azathioprine, cyclosporine, and corticosteroids in Behçet's disease: An openlabel trial. Arthritis \& Rheumatism, 52, 2478-2484. doi:10.1002/art.21231

[12] Russell, A.I., Lawson, W.A. and Haskard, D.O. (2001) 
Potential new therapeutic options in Behçet's syndrome. BioDrugs, 15, 25-35. doi:10.2165/00063030-200115010-00003

[13] Pato, E., Munoz-Fernandez, S., Francisco, F., Abad, M.A., Maese, J., Ortiz, A., et al. (2011) Systematic review on the effectiveness of immunosuppressants and biological therapies in the treatment of autoimmune posterior uveitis. Seminars in Arthritis and Rheumatism, 40, 314-323. doi:10.1016/j.semarthrit.2010.05.008

[14] Evereklioglu, C. (2011) Ocular Behcet disease: current therapeutic approaches. Current Opinion in Ophthalmology, 22, 508-516. doi:10.1097/ICU.0b013e32834bbe91

[15] Handa, T., Tsunekawa, H., Yoneda, M., Watanabe, D., Mukai, T., Yamamura, M., et al. (2011) Long-term remission of ocular and extraocular manifestations in Behçet's disease using infliximab. Clinical and Experimental Rheumatology, 29, S58-63.

[16] Keino, H., Watanabe, T., Taki, W. and Okada, A.A. (2011) Effect of infliximab on gene expression profiling in Behçet's disease. Investigative Ophthalmology \& Visual Science, 52, 7681-7686. doi:10.1167/iovs.11-7999

[17] Keino, H., Okada, A.A., Watanabe, T. and Taki, W. (2011) Decreased ocular inflammatory attacks and background retinal and disc vascular leakage in patients with Behçet's disease on infliximab therapy. British Journal of Ophthalmology, 95, 1245-1250. doi:10.1136/bjo.2010.194464

[18] Okada, A.A., Goto, H., Ohno, S. and Mochizuki, M. (2012) Multicenter study of infliximab for refractory uveoretinitis in Behcet disease. Archives of Ophthalmology, 130, 592-598. doi:10.1001/archophthalmol.2011.2698

[19] Cantini, F., Niccoli, L., Nannini, C., Kaloudi, O., Cassara, E., Susini, M., et al. (2012) Efficacy of infliximab in refractory Behçet's disease-associated and idiopathic posterior segment uveitis: A prospective, follow-up study of 50 patients. Biologics, 6, 5-12.

[20] Ito, T., Sonoda, K.H., Hijioka, K., Fujimoto, T. and Ishibashi, T. (2010) Acquired resistance to infliximab against uveitis due to Behçet's disease after one year of administration. Japanese Journal of Ophthalmology, 54, 502504. doi:10.1007/s10384-010-0859-0

[21] Suhler, E.B., Smith, J.R., Wertheim, M.S., Lauer, A.K., Kurz, D.E., Pickard, T.D., et al. (2005) A prospective trial of infliximab therapy for refractory uveitis: Preliminary safety and efficacy outcomes. Archives of Ophthalmology, 123, 903-912. doi:10.1001/archopht.123.7.903

[22] Benitah, N.R., Sobrin, L. and Papaliodis, G.N. (2011) The use of biologic agents in the treatment of ocular manifestations of Behçet's disease. Seminars in Ophthalmology, 26, 295-303. doi:10.3109/08820538.2011.588665

[23] Fries, W., Giofre, M.R., Catanoso, M. and Lo Gullo, R. (2002) Treatment of acute uveitis associated with Crohn's disease and sacroileitis with infliximab. The American Journal of Gastroenterology, 97, 499-500. doi:10.1111/j.1572-0241.2002.05514.x

[24] Ally, M.R., Veerappan, G.R. and Koff, J.M. (2008) Treatment of recurrent Crohn's uveitis with infliximab. The American Journal of Gastroenterology, 103, 2150-
2151. doi:10.1111/j.1572-0241.2008.01982_12.x

[25] Kruithof, E., Kestelyn, P., Elewaut, C., Elewaut, D., Van Den Bosch, F., Mielants, H., et al. (2002) Successful use of infliximab in a patient with treatment resistant spondyloarthropathy related uveitis. Annals of the Rheumatic Diseases, 61, 470. doi:10.1136/ard.61.5.470

[26] Baraliakos, X., van den Berg, R., Braun, J. and van der Heijde, D. (2012) Update of the literature review on treatment with biologics as a basis for the first update of the ASAS/EULAR management recommendations of ankylosing spondylitis. Rheumatology (Oxford), 51, 13781387. doi:10.1093/rheumatology/kes026

[27] El Maghraoui, A. (2011) Extra-articular manifestations of ankylosing spondylitis: Prevalence, characteristics and therapeutic implications. European Journal of Internal Medicine, 22, 554-560. doi:10.1016/j.ejim.2011.06.006

[28] Mangge, H., Heinzl, B., Grubbauer, H.M., El-Shabrawi, Y. and Schauenstein, K. (2003) Therapeutic experience with infliximab in a patient with polyarticular juvenile idiopathic arthritis and uveitis. Rheumatology International, 23, 258-261. doi:10.1007/s00296-003-0308-1

[29] Sharma, S.M., Ramanan, A.V., Riley, P. and Dick, A.D. (2007) Use of infliximab in juvenile onset rheumatological disease-associated refractory uveitis: Efficacy in joint and ocular disease. Annals of the Rheumatic Diseases, 66, 840-841. doi:10.1136/ard.2006.065441

[30] Khalifa, Y.M., Bailony, M.R. and Acharya, N.R. (2010) Treatment of pediatric vogt-koyanagi-harada syndrome with infliximab. Ocular Immunology and Inflammation, 18, 218-222. doi:10.3109/09273941003739910

[31] Sukumaran, S., Marzan, K., Shaham, B. and Reiff, A. (2012) High dose infliximab in the treatment of refractory uveitis: Does dose matter? ISRN Rheumatology, 2012, 765380.

[32] Farvardin, M., Afarid, M. and Shahrzad, S. (2012) Longterm effects of intravitreal infliximab for treatment of sight-threatening chronic noninfectious uveitis. Journal of Ocular Pharmacology and Therapeutics, 28, 628-631. doi:10.1089/jop.2011.0199

[33] Markomichelakis, N., Delicha, E., Masselos, S. and Sfikakis, P.P. (2012) Intravitreal infliximab for sight-threatening relapsing uveitis in behcet disease: A pilot study in 15 patients. American Journal of Ophthalmology, 154, 534-541. e1. doi:10.1016/j.ajo.2012.03.035

[34] Rudwaleit, M., Rodevand, E., Holck, P., Vanhoof, J., Kron, M., Kary, S., et al. (2009) Adalimumab effectively reduces the rate of anterior uveitis flares in patients with active ankylosing spondylitis: Results of a prospective open-label study. Annals of the Rheumatic Diseases, 68 , 696-701. doi:10.1136/ard.2008.092585

[35] Mushtaq, B., Saeed, T., Situnayake, R.D. and Murray, P.I. (2007) Adalimumab for sight-threatening uveitis in Behçet's disease. Eye (Lond), 21, 824-825. doi:10.1038/sj.eye. 6702352

[36] Takase, K., Ohno, S., Ideguchi, H., Uchio, E., Takeno, M. and Ishigatsubo, Y. (2011) Successful switching to adalimumab in an infliximab-allergic patient with severe Behcet disease-related uveitis. Rheumatology International, 31, 243-245. doi:10.1007/s00296-009-1178-y 
[37] Kotaniemi, K., Saila, H. and Kautiainen, H. (2011) Longterm efficacy of adalimumab in the treatment of uveitis associated with juvenile idiopathic arthritis. Clinical Ophthalmology, 5, 1425-1429. doi:10.2147/OPTH.S23646

[38] Erckens, R.J., Mostard, R.L., Wijnen, P.A., Schouten, J.S. and Drent, M. (2012) Adalimumab successful in sarcoidosis patients with refractory chronic non-infectious uveitis. Graefe's Archive for Clinical and Experimental Ophthalmology, 250, 713-720. doi:10.1007/s00417-011-1844-0

[39] Diaz-Llopis, M., Salom, D., Garcia-de-Vicuna, C., Cordero-Coma, M., Ortega, G., Ortego, N., et al. (2012) Treatment of refractory uveitis with adalimumab: A prospective multicenter study of 131 patients. Ophthalmology, 119, 1575-1581.

[40] Leccese, P., Latanza, L., D’Angelo, S., Padula, A. and Olivieri, I. (2011) Efficacy of switching to adalimumab in a patient with refractory uveitis of Behçet's disease to infliximab. Clinical and Experimental Rheumatology, 29, S93.

[41] Bawazeer, A., Raffa, L.H. and Nizamuddin, S.H. (2010) Clinical experience with adalimumab in the treatment of ocular Behcet disease. Ocular Immunology and Inflammation, 18, 226-232. doi:10.3109/09273948.2010.483314

[42] Dhingra, N., Morgan, J. and Dick, A.D. (2009) Switching biologic agents for uveitis. Eye, 23, 1868-1870. doi:10.1038/eye.2009.203

[43] Reiff, A., Takei, S., Sadeghi, S., Stout, A., Shaham, B., Bernstein, B., et al. (2001) Etanercept therapy in children with treatment-resistant uveitis. Arthritis \& Rheumatism, 44, 1411-1415. doi:10.1002/1529-0131(200106)44:6<1411::AID-ART23 5>3.0.CO, 2-O

[44] Quartier, P., Taupin, P., Bourdeaut, F., Lemelle, I., Pillet, P., Bost, M., et al. (2003) Efficacy of etanercept for the treatment of juvenile idiopathic arthritis according to the onset type. Arthritis \& Rheumatism, 48, 1093-1101. doi:10.1002/art.10885

[45] Reddy, A.R. and Backhouse, O.C. (2003) Does etanercept induce uveitis? British Journal of Ophthalmology, 87, 925. doi:10.1136/bjo.87.7.925

[46] Reiff, A. (2003) Long-term outcome of etanercept therapy in children with treatment-refractory uveitis. Arthritis \& Rheumatism, 48, 2079-2080.doi:10.1002/art.11155

[47] Schmeling, H. and Horneff, G. (2005) Etanercept and uveitis in patients with juvenile idiopathic arthritis. Rheumatology, 44, 1008-1011. doi:10.1093/rheumatology/keh658

[48] Taban, M., Dupps, W.J., Mandell, B. and Perez, V.L. (2006) Etanercept (enbrel)-associated inflammatory eye disease: Case report and review of the literature. Ocular Immunology and Inflammation, 14, 145-150. doi:10.1080/09273940600659393

[49] Lim, L.L., Fraunfelder, F.W. and Rosenbaum, J.T. (2007) Do tumor necrosis factor inhibitors cause uveitis? A registry-based study. Arthritis \& Rheumatism, 56, 32483252. doi:10.1002/art.22918

[50] Gaujoux-Viala, C., Giampietro, C., Gaujoux, T., Ea, H.K.,
Prati, C., Orcel, P., et al. (2012) Scleritis: A paradoxical effect of etanercept? Etanercept-associated inflammatory eye disease. The Journal of Rheumatology, 39, 233-239. doi:10.3899/jrheum.110865

[51] Schafer, V.S. and Zwerina, J. (2012) Biologic treatment of large-vessel vasculitides. Current Opinion in Rheumatology, 24, 31-37. doi:10.1097/BOR.0b013e32834dc392

[52] Muselier, A., Bielefeld, P., Bidot, S., Vinit, J., Besancenot, J.F. and Bron, A. (2011) Efficacy of tocilizumab in two patients with anti-TNF-alpha refractory uveitis. Ocular Immunology and Inflammation, 19, 382-383. doi:10.3109/09273948.2011.606593

[53] Shapiro, L.S., Farrell, J. and Haghighi, A.B. (2012) Tocilizumab treatment for neuro-Behçet's disease, the first report. Clinical Neurology and Neurosurgery, 114, 297 298. doi:10.1016/i.clineuro.2011.10.024

[54] Tappeiner, C., Heinz, C., Ganser, G. and Heiligenhaus, A. (2012) Is tocilizumab an effective option for treatment of refractory uveitis associated with juvenile idiopathic arthritis? The Journal of Rheumatology, 39, 1294-1295. doi:10.3899/jrheum.120010

[55] William, M., Faez, S., Papaliodis, G.N. and Lobo, A.M. (2012) Golimumab for the treatment of refractory juvenile idiopathic arthritis-associated uveitis. Journal of Ophthalmic Inflammation and Infection, 2, 231-233.

[56] Planck, S.R., Woods, A., Clowers, J.S., Nicklin, M.J., Rosenbaum, J.T. and Rosenzweig, H.L. (2012) Impact of IL-1 signalling on experimental uveitis and arthritis. Annals of the Rheumatic Diseases, 71, 753-760. doi:10.1136/annrheumdis-2011-200556

[57] Teoh, S.C., Sharma, S., Hogan, A., Lee, R., Ramanan, A.V. and Dick, A.D. (2007) Tailoring biological treatment: Anakinra treatment of posterior uveitis associated with the CINCA syndrome. British Journal of Ophthalmology, 91, 263-264.

[58] Gul, A., Tugal-Tutkun, I., Dinarello, C.A., Reznikov, L., Esen, B.A., Mirza, A., et al. (2012) Interleukin-1betaregulating antibody XOMA 052 (gevokizumab) in the treatment of acute exacerbations of resistant uveitis of Behçet's disease: An open-label pilot study. Annals of the Rheumatic Diseases, 71, 563-566. doi:10.1136/annrheumdis-2011-155143

[59] Smith, K.G., Jones, R.B., Burns, S.M. and Jayne, D.R. (2006) Long-term comparison of rituximab treatment for refractory systemic lupus erythematosus and vasculitis: Remission, relapse, and re-treatment. Arthritis \& Rheumatism, 54, 2970-2982. doi:10.1002/art.22046

[60] Meijer, J.M., Pijpe, J., Vissink, A., Kallenberg, C.G. and Bootsma, H. (2009) Treatment of primary Sjögren syndrome with rituximab: Extended follow-up, safety and efficacy of retreatment. Annals of the Rheumatic Diseases, 68, 284-285. doi:10.1136/ard.2008.092601

[61] Smith, R.M., Jones, R.B., Guerry, M.J., Laurino, S., Catapano, F., Chaudhry, A., et al. (2012) Rituximab for remission maintenance in relapsing ANCA-associated vasculitis. Arthritis \& Rheumatism, 64, 3760-3769. doi:10.1002/art.34583

[62] Nakken, B., Munthe, L.A., Konttinen, Y.T., Sandberg, A.K., Szekanecz, Z., Alex, P., et al. (2011) B-cells and 
their targeting in rheumatoid arthritis-Current concepts and future perspectives. Autoimmunity Reviews, 11, 28-34. doi:10.1016/j.autrev.2011.06.010

[63] Tappeiner, C., Heinz, C., Specker, C. and Heiligenhaus, A. (2007) Rituximab as a treatment option for refractory endogenous anterior uveitis. Ophthalmic Research, 39, 184-186. doi:10.1159/000103239

[64] Davatchi, F., Shams, H., Rezaipoor, M., Sadeghi-Abdollahi, B., Shahram, F., Nadji, A., et al. (2010) Rituximab in intractable ocular lesions of Behçet's disease; randomized single-blind control study (pilot study). International Journal of Rheumatic Diseases, 13, 246-252. doi:10.1111/j.1756-185X.2010.01546.X
[65] Heiligenhaus, A., Miserocchi, E., Heinz, C., Gerloni, V. and Kotaniemi, K. (2011) Treatment of severe uveitis associated with juvenile idiopathic arthritis with anti-CD20 monoclonal antibody (rituximab). Rheumatology, 50, 1390-1394.doi:10.1093/rheumatology/ker107

[66] Miserocchi, E. and Modorati, G. (2012) Rituximab for noninfectious uveitis. Developments in Ophthalmology, 51, 98-109. doi:10.1159/000336188

[67] Zulian, F., Balzarin, M., Falcini, F., Martini, G., Alessio, M., Cimaz, R., et al. (2010) Abatacept for severe antitumor necrosis factor alpha refractory juvenile idiopathic arthritis-related uveitis. Arthritis Care \& Research, 62 821-825. doi:10.1002/acr.20115

\section{MAIN ABBREVIATIONS}

TNF: tumor necrosis factor;

VA: visual acuity;

JIA: juvenile idiopathic arthritis;

RA: rheumatoid arthritis;

AS: ankylosing spondylitis;

MTX: methotrexate;

DMARD: disease-modifying antirheumatic drug. 